\title{
First experiences with Lu-177 PSMA therapy in combination with Pembrolizumab or after pretreatment with Olaparib in single patients
}

Vikas Prasad ${ }^{1 \S}$, Friedemann Zengerling ${ }^{2}$, Jochen P. Steinacker ${ }^{1}$, Christian Bolenz $^{2}$, Meinrad Beer ${ }^{3}$, Thomas Wiegel ${ }^{4}$, Matthias Eiber ${ }^{5}$, Neil Fleshner ${ }^{6}$, Ambros J. Beer ${ }^{1}$

1) Department of Nuclear Medicine, Ulm University Hospital , Germany

2) Department of Urology, Ulm University Hospital , Germany

3) Department of Radiology, Ulm University Hospital , Germany

4) Department of Radiation Oncology, Ulm University Hospital , Germany

5) Department of Nuclear Medicine, Technical University of Munich, Germany

6) Division of Urology, University of Toronto, Canada

\section{§Corresponding Author:}

Vikas Prasad

Department of Nuclear Medicine, Ulm University Hospital, Germany

Email: vikas.prasad@uniklinik-ulm.de 


\section{ABSTRACT}

Synergistic effects of immunotherapy with pembrolizumab or drugs targeting DNA damage e.g. olaparib could be used to overcome the limitations of radioligand therapy (RLT) with Lu-177 prostate specific membrane antigen (PSMA) in metastasized castrate resistant prostate cancer (mCRPC) patients. Here, we present two patients receiving such combination / sequential therapies. Methods: RLT was performed at 6-8 week intervals after they either exhausted or were considered unfit for all approved conventional treatment. Patient 1 was on pembrolizumab for his squamous cell carcinoma of the skin whereas patient 2 received RLT sequentially 4 weeks after a 3 months monotherapy with olaparib. Results: Both patients tolerated RLT without any significant hematotoxicity. Patient 2 showed radiological and biochemical response whereas patient 1 achieved PSA stabilization after 3 therapy cycles. Conclusion: These cases indicate that RLT in combination with pembrolizumab or sequentially after olaparib might be well tolerated in single patients.

Key words: PSMA; Lu-177, pembrolizumab, olaparib

Running Title: RLT together with Pembrolizumab or Olaparib in PCa 


\section{INTRODUCTION}

PSMA targeting RLT for mCRPC shows good therapeutic efficacy in $1 / 3^{\text {rd }}$ of the patients whereas approximately $1 / 3^{\text {rd }}$ are non-responders despite showing intense PSMA expression on positron emission tomography (PET) (1,2). Additional to PSMA heterogeneity, differences in tumour biology and response to internal irradiation can explain this heterogeneity in therapeutic efficacy of RLT (3). Hence there is a need for new strategies to augment the therapeutic efficacy of RLT. In a phase II trial, pembrolizumab has shown promising clinical activity in mCRPC (4). Similarly, the PROfound study has shown the efficacy of olaparib in mCRPC with DNA damage response (DDR) gene aberrations (5). RLT together with pembrolizumab or sequentially after olaparib might have synergistic effects, however there are little data on the tolerability of such combined treatments. Here, we present two cases which showed good tolerability of such combination / sequential therapies.

\section{MATERIALS AND METHODS}

\section{Patient Characteristics}

Two out of 69 prostate cancer patients receiving RLT between 01/2018 and 06/2020 were treated either sequentially or in combination with olaparib or pembrolizumab. The institutional review board (IRB or equivalent) approved this retrospective case series study and all subjects signed a written informed consent. Both mCRPC patients were in an advanced stage with no conventional therapeutic options left. RLT was offered to the patients after discussing the therapeutic strategy in an interdisciplinary tumor board. 
Radiopharmaceuticals and Imaging

Lu-177 was acquired from ITM (Garching, Germany). GMP grade PSMA-11, PSMA-617 and PSMA-I\&T were acquired from Endocyte/ABX (Radeberg, Germany) Scintomics (Fuerstenfeldbruck, Germany), respectively. Radiolabeling was performed according to the established procedure in GMP certified radiopharmacy laboratory (6). PSMA-ligand PET pre and post-therapy was performed as previously described $(7,8)$.

\section{RESULTS}

Clinical Course of Patient 1 (Figure 1)

A 90 years old patient was first diagnosed with Gleason Score 4+3 PCa in 2007. Because of his advanced age he was kept under watchful waiting and biopsy was renewed in 2013, which revealed status quo. However, because of rising PSA, bone scan was performed in 2014, which showed bone metastases resulting in treatment with leuprolide. In 08/2018 he developed mCRPC for which he was placed on enzalutamide. In 09/2019 together with rising PSA, bone scan and CT scan revealed progressive asymptomatic osteoblastic bone lesions. Due to his advanced stage, the patient was considered unfit for chemotherapy and due to lack of symptoms from his bone metastases, no Ra-223 treatment was offered. Hence the patient underwent PSMA PET/CT, which revealed intense uptake of PSMA in bone lesions and was referred to RLT. At the time of RLT he was on treatment with 3 weekly pembrolizumab $200 \mathrm{mg}$ for a second malignancy (locally advanced cutaneous squamous cell carcinoma, SCC of forehead region). He was treated with 5 GBq Lu-177 PSMA per cycle (first two cycles with Lu-177 PSMA 617 and third cycle with Lu-177 PSMA IT). The reason for performing 2 cycles with Lu-177 PSMA 617 and one with Lu-177 PSMA IT was purely logistical and 
based on the availability of the peptides. His ECOG 1 status remained unchanged after the therapy. Hematological toxicity profile revealed no significant changes in the erythrocyte (Ery), thrombocyte (thrombo), hemoglobin $(\mathrm{Hb})$ and leucocyte (leuco) counts (Figure 2). His renal function remained unchanged. Overall, the post therapy scan revealed stable to mild decreasing PSMA uptake in known bone lesions without any evidence of new foci. The SCC showed no PSMA uptake in the post therapy scan. His PSA declined by $40 \%$.

Clinical Course of Patient 2 (Figure 3)

A 71 years old patient, was first diagnosed with cT3 pN+cM0, GS 4+4 PCa (iPSA $521.6 \mathrm{ng} / \mathrm{ml}$ ) in 04/2008. At initial diagnosis, he was 59 years old and was offered pelvic lymphadenectomy and prostatectomy. Intraoperatively, it was observed that the primary tumor was inoperable, so consequently only pelvic lymphadenectomy was performed. Afterwards, starting from 08/2008 till 01/2017, he was put on zoladex, trenantone, pamorelin, bicalutamid followed by enzalutamide (mCRPC in 08/2015). Unfortunately, he became progressive (biochemically and radiologically) in 02/2017 leading to treatment with docetaxel, which had to be terminated prematurely due to severe allergic reactions. Thus the patient was evaluated for further treatment options and enrolled in the Keynote365 (olaparib + pembrolizumab) study. Despite showing excellent biochemical and radiological response, in $11 / 2017$ he had to be taken out of the study due to significant pembrolizumab-related adverse events including immune-related arthritis and hypophysitis. PSA- and imaging findings remained stable until 02/2019 when he developed pain in the sternum. On a CT scan disease progression was confirmed. For symptom control, his sternal metastasis was locally irradiated and considering his 
excellent response in the Keynote-365 trial olaparib monotherapy was started afterwards. Pembrolizumab could not be added because of known severe side effects. Without responding to olaparib re-challenge the patient showed PSA progression in 08/2019, so he was referred for RLT. RLT was started 4 weeks after the last dose of Olaparib. As shown in figure 4 , he had excellent persistent response to RLT even at 16 weeks post $4^{\text {th }}$ RLT. His laboratory parameters showed no significant bone marrow toxicity whereas his renal function also remained unchanged compared to the baseline (Figure 4).

\section{DISCUSSION}

In this report we have shown two mCRPC patients with good tolerance of Lu-177 PSMA RLT in combination with pembrolizumab or sequentially after olaparib, which in the future might be an interesting option to improve therapeutic efficacy and / or overcome resistance to $\mathrm{RLT}$.

One reason for an insufficient response to RLT in mCRPC patients might be spatial and temporal heterogeneity of PSMA expression. It is well known that PSMA expression is upregulated in most but not all PCs $(9,10)$. Even intensely PSMA-expressing lesions on PSMA PET often show insufficient response to Lu-177 PSMA RLT. The level of PSMA expression is only one of many factors influencing responsiveness to RLT. Other factors like DNA repair mechanism or immune phenomena probably also play a crucial role in resistance to Lu-177 PSMA RLT. One of the proposed strategies to potentiate the efficacy of RLT is by combining or sequencing it with other targeted agents.

Selecting the right drug to combine with RLT is a challenge considering the fact that all targeted drugs have their own side effects. Tumor - internal radiation interaction takes place at the intracellular and extracellular level. For inducing durable response 
sufficient double strand (ds) breakage in DNA is needed (11). There are two pathways in which cells react to radiation: homologous recombination (HR) and non-homologous end joining (NHEJ) (12). Poly (ADP-ribose) polymerase is involved in this repair process. Drugs inhibiting this pathway may increase radiation sensitivity. Recently olaparib, a PARP inhibitor was approved for patients with prostate cancer harboring DNA damage repair gene aberrations. That is why combining RLT with PARP inhibitor appears to be an attractive option. However a combination may not be without toxicity. In our series, patient 2 was treated sequentially with RLT after olaparib. As olaparib can be hematotoxic, we allowed sufficient wash out time and hence treated the patient with RLT 4 weeks after the last dose of olaparib. Indeed, the follow-up hematological parameters showed that the 4 weeks washout period does not lead to significant hematotoxicity whereas the RLT efficacy seemed to be maintained. It is difficult to say if the good response seen in this patient was RLT related or due to the synergistic effects of olaparib.

While the intracellular interaction with DNA is central to the therapeutic efficacy $R L T$, its potential positive effect on tumor immunity makes RLT extremely attractive for combination immunotherapy $(13,14)$. Patient 1 was on pembrolizumab due to cutaneous SCC. It was unclear whether RLT in combination with immunotherapy would be well tolerated, especially considering the patients' age of 90 years. Additionally, there was no data at the time of the RLT on the optimum dosage of pembrolizumab and RLT without inducing toxicity while maintaining individual therapeutic efficacy. This case showed that RLT with $5 \mathrm{GBq}$ in combination with 3-weekly $200 \mathrm{mg}$ pembrolizumab can be well tolerated. The therapeutic efficacy was adequate showing a biochemical response with a PSA-decline of $40 \%$. 
One of the major limitations, apart from low number of patients is that we do not have information on the DDR or PD-1 status of the lesions. Olaparib has been shown to increase OS and PFS in patients harboring DDR mutations and as lesions harboring DDR expression show higher PSMA expression, it is important to perform lesion biopsies prior to such sequential or combination therapies to assess the DDR status. Similarly PD-1 positivity can help in appropriate patient selection for immunotherapy. However we do not think these limitations diminish the importance of these two cases as the primary aim of this manuscript is to highlight that sequential olaparib or combined pembrolizumab with RLT is well tolerated. Ongoing prospective phase 1/2 studies (ClinicalTrials.gov Identifier: NCT03874884; NCT03805594) will provide more data on RLT in combination with pembrolizumab or olaparib.

\section{CONCLUSIONS}

These two cases indicate that RLT in combination with pembrolizumab or sequentially after olaparib might be well tolerated in single patients. Further studies in larger patient populations are needed to assess the safety and efficacy of DNA repair gene targeting drugs and immunotherapy in combination with RLT. 


\section{Acknowledgements:}

$\mathrm{CB}, \mathrm{MB}, \mathrm{FZ}$ and $\mathrm{AB}$ are part of the $\mathrm{i}^{2} \mathrm{SOUL}$ consortium at Ulm University Hospital, which we thank for support.

\section{Conflict of Interest}

VP is scientific advisor of Point Biopharma and has acted as consulted for ITM. NF is a co-founder of Point Biopharma. AB has acted as consultant for Siemens. CB discloses about speaking fees and sponsored research from AstraZeneca. FZ has advisory role for MSD Sharp \& Dohme. ME received funding from the Deutsche Forschungsgemeinschaft within project B11. MB, TW, and SJP declare no potential conflicts of interest relevant to this article exist. No other potential conflicts of interest relevant to this article exist.

\section{Key Points}

Question: Is it feasible to perform RLT with Lu-177 PSMA in mCRPC patients in combination or sequentially with pembrolizumab or olaparib?

Pertinent Findings: In single patients, RLT in combination with pembrolizumab or sequentially to olaparib might be well tolerated.

Implications for patient care: In selected patients with $\mathrm{mCRPC}$, patients on pembrolizumab or after olaparib can be treated with RLT. 


\section{REFERENCES:}

1) Ravi Kumar AS, Hofman MS. Mechanistic insights for optimising PSMA radioligand therapy. Clin Cancer Res. 2020 [Epub ahead of print ]

2) Miyahira AK, Pienta KJ, Morris MJ, et al. Meeting report from the Prostate Cancer Foundation PSMA-directed radionuclide scientific working group. Prostate. 2018;78:775-789.

3) Weichselbaum RR, Liang H, Deng L, Fu YX. Radiotherapy and immunotherapy: a beneficial liaison? Nat Rev Clin Oncol. 2017;14:365-379.

4) Antonarakis ES, Piulats JM, Gross-Goupil M, et al. Pembrolizumab for treatmentrefractory metastatic castration-resistant prostate cancer: multicohort, open-label Phase II KEYNOTE-199 Study. J Clin Oncol. 2020; 38:395-405.

5) De Bono J, Mateo J, Fizazi K, et al. Olaparib for metastatic castration-resistant prostate cancer. N Engl J Med. 2020 [Epub ahead of print ].

6) Delker A, Fendler WP, Kratochwil C, et al. Dosimetry for (177)Lu-DKFZ-PSMA617: a new radiopharmaceutical for the treatment of metastatic prostate cancer. Eur J Nucl Med Mol Imaging. 2016;43:42-51.

7) Fendler WP, Eiber M, Beheshti M, et al. ${ }^{68} \mathrm{Ga}-\mathrm{PSMA}$ PET/CT: Joint EANM and SNMMI procedure guideline for prostate cancer imaging: version 1.0. Eur $\mathrm{J} \mathrm{NuCl}$ Med Mol Imaging. 2017;44:1014-1024.

8) Szabo Z, Mena E, Rowe SP, et al. Initial evaluation of [18F]DCFPyL for ProstateSpecific Membrane Antigen (PSMA)-targeted PET imaging of prostate cancer. Mol Imaging Biol. 2015;17:565-574

9) Paschalis A, Sheehan B, Riisnaes R, et al. Prostate-specific Membrane Antigen heterogeneity and DNA repair defects in prostate cancer. European Urology. 2019;76:469-478

10)Damjanovic J, Janssen JC, Prasad V, et al. 68Ga-PSMA-PET/CT for the evaluation of liver metastases in patients with prostate cancer. Cancer Imaging. 2019; 19:37.

11)O'Driscoll M, Jeggo PA: The role of double-strand break repair - insights from human genetics. Nat Rev Cancer. 2006;7:45-54

12) Haber JE. Partners and pathways repairing a double-strand break. Trends Genet. 2000;16:259-264

13)Demaria $\mathrm{S}, \mathrm{Ng} \mathrm{B}$, Devitt $\mathrm{ML}$, et al. lonizing radiation inhibition of distant untreated tumors (abscopal effect) is immune mediated. Int $J$ Radiat Oncol Biol Phys. 2004;58:862-70

14)Wu $Y$, Pfeifer AK, Myschetzky R, et al. Induction of anti-tumor immune responses by peptide receptor radionuclide therapy with $177 \mathrm{Lu}$-DOTATATE in a murine model of a human neuroendocrine tumor. Diagnostics. 2013;3,344-355 


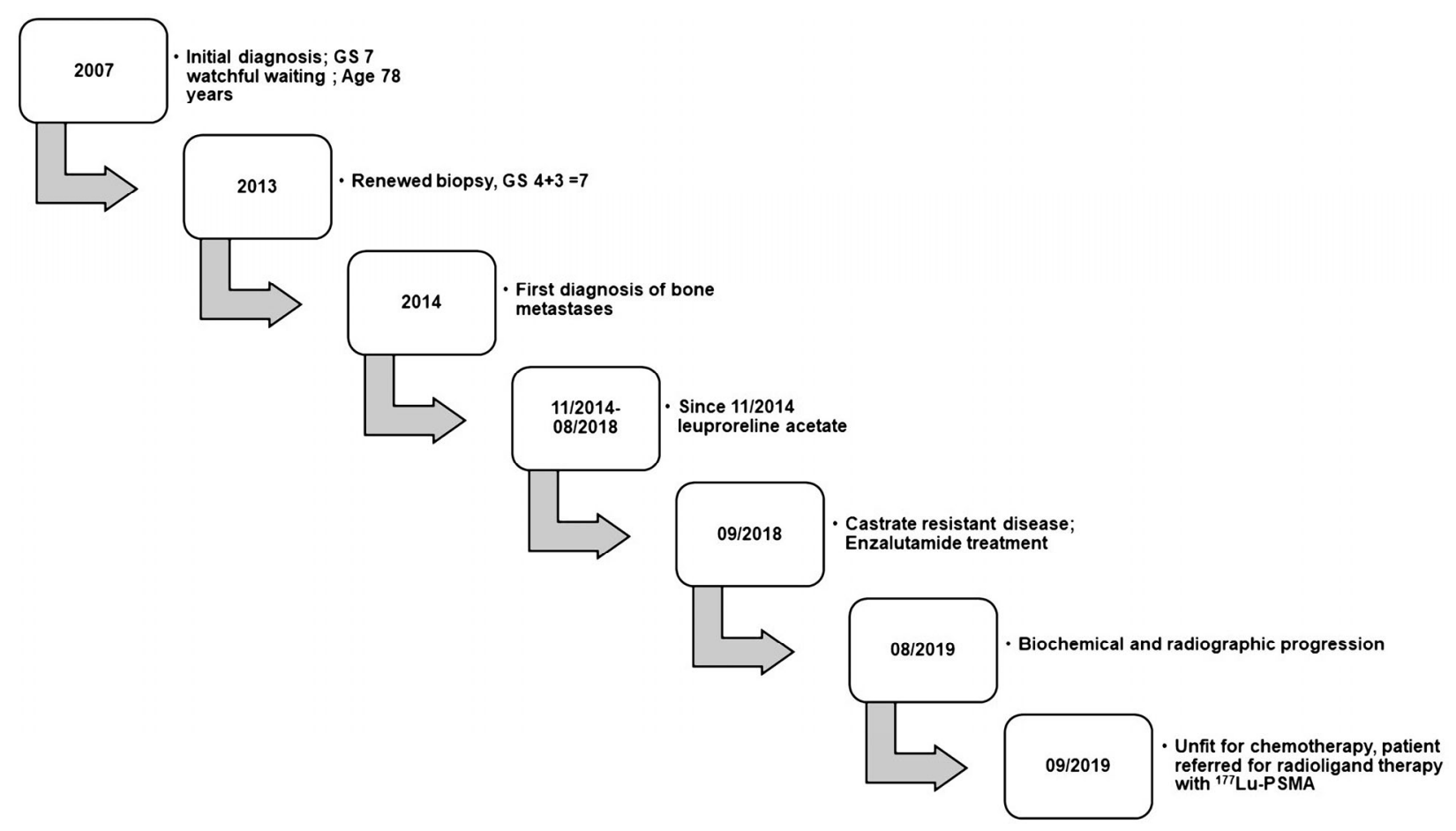

Figure 1: Clinical course of patient 1 

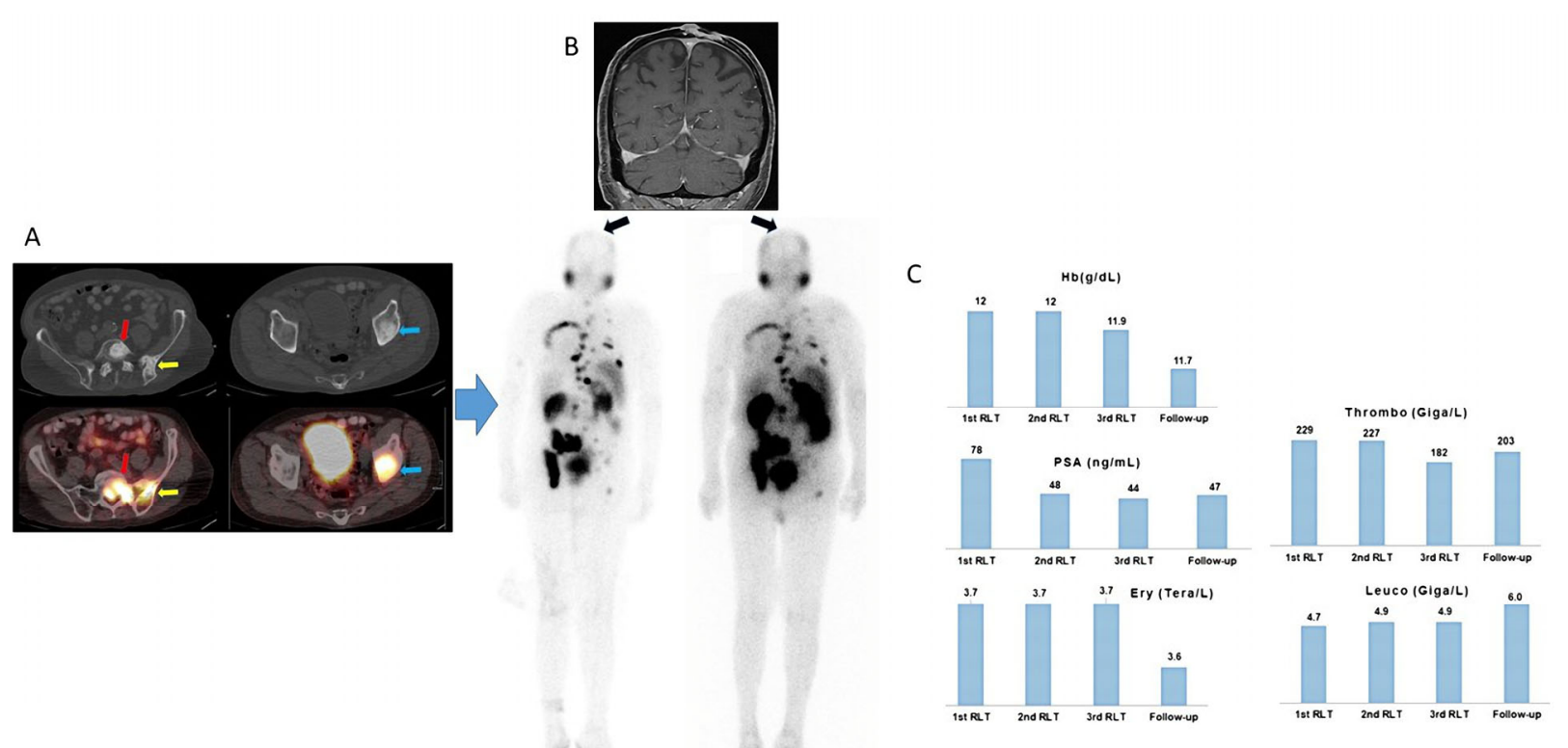

Figure 2: Posterior view (left after 1st therapy) of post-treatment scintigraphy showing intense radiotracer uptake in the known bone lesions (ribs, sternum, thoracic and lumbar vertebrae, sacrum, and iliac bones); mild to moderate reduction in intensity of uptake was observed in post-treatment scan after 3rd therapy cycle (right). A coronal MRI, T1w sequence $(B)$ at start of 1 st RLT shows the squamous cell carcinoma in the parietal region of the skull; this lesion showed no PSMA uptake in post therapy scans. A) axial section of CT (upper panel) and fused PET/CT (lower panel) demonstrate intense F-18 DCFPyL uptake (lower panel) of osteoblastic bone lesions in the $1^{\text {st }}$ sacral vertebra (red arrow), left iliac bone / iliosacral joint (yellow arrow) and left acetabulum (blue arrow). C) Sequential lab tests showed a PSA-decline from 78 to $47 \mathrm{ng} / \mathrm{ml}$, but no substantial changes in hematological parameters. 


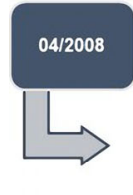

Initial diagnosis; Initial tumor

Stage $\mathrm{cT} 3$, pN+, cM0
GS $4+4=8$, iPSA 521.6

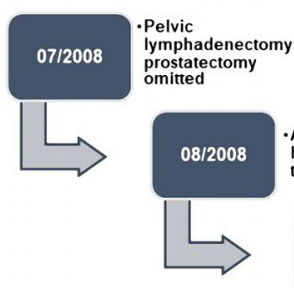

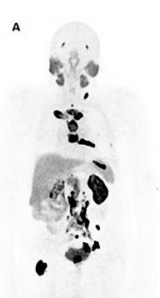

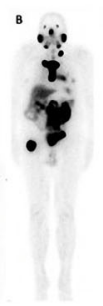

T (zoledronic acid,

ADT (zoledronic acid,

leuproreline acetate
triptorelin, Bicalutamid)

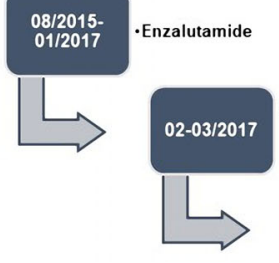

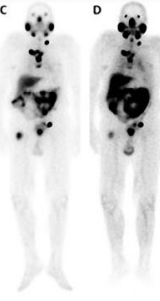
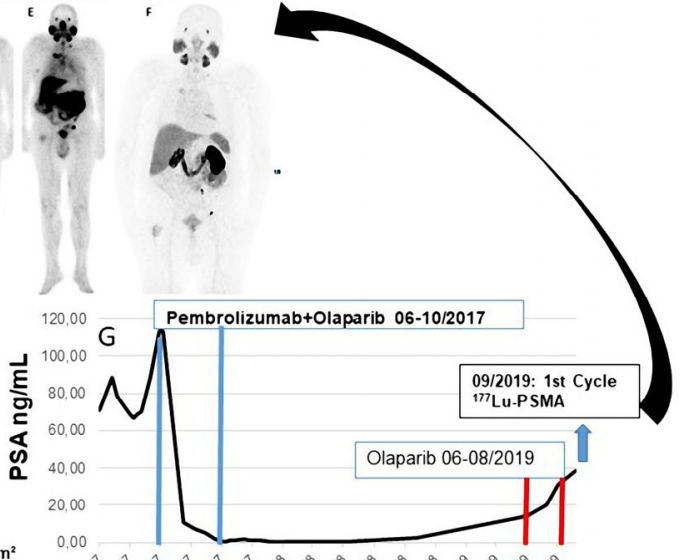

2 cycles docetaxel $75 \mathrm{mg} / \mathrm{m}^{2}$ ter meekly, premature reaction

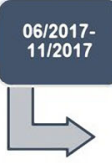

-Keynote-365 (Olaparib+Pembrolizumab); DDR expression unknown; patient achieved partial remission. However he also developed

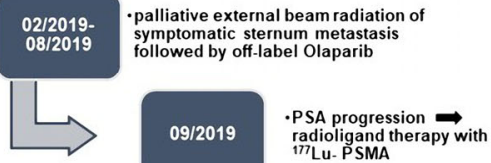

Figure 3: PSMA PET/CT maximum intensity projection images ( $A$ and $F$ ) and post therapy Lu-177 PSMA scintigraphy images (B-E). G) PSA follow-up of the patient 2 staring from 02/2017. The chart is showing the clinical course of the disease. 


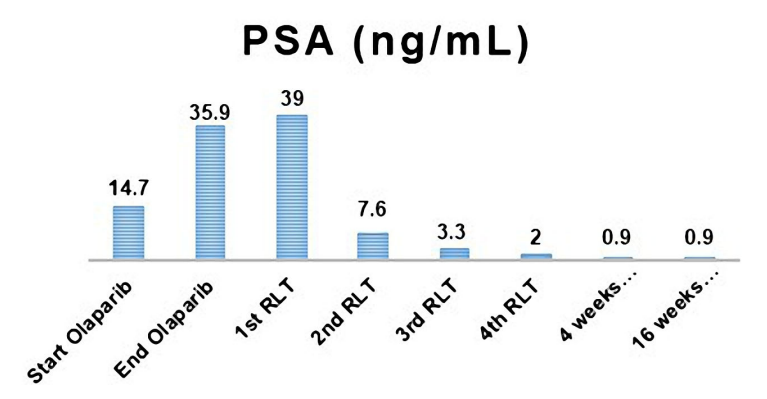

Thrombo (Giga/L)

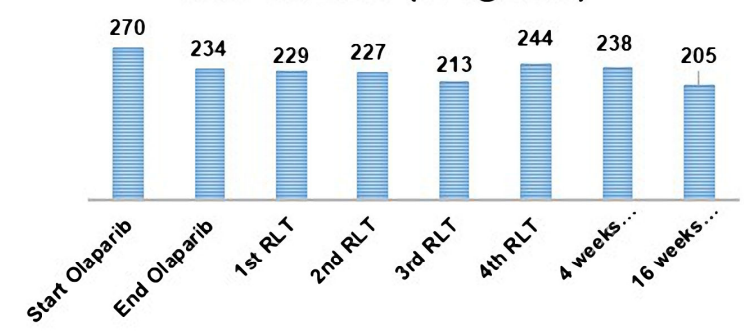

$\mathrm{Hb}(\mathrm{g} / \mathrm{dL})$

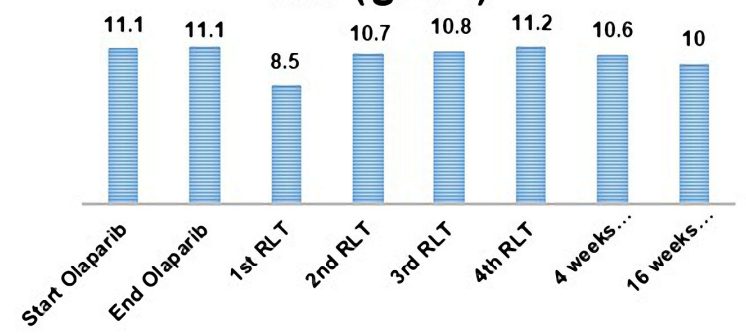

\section{Ery (Tera/L)}
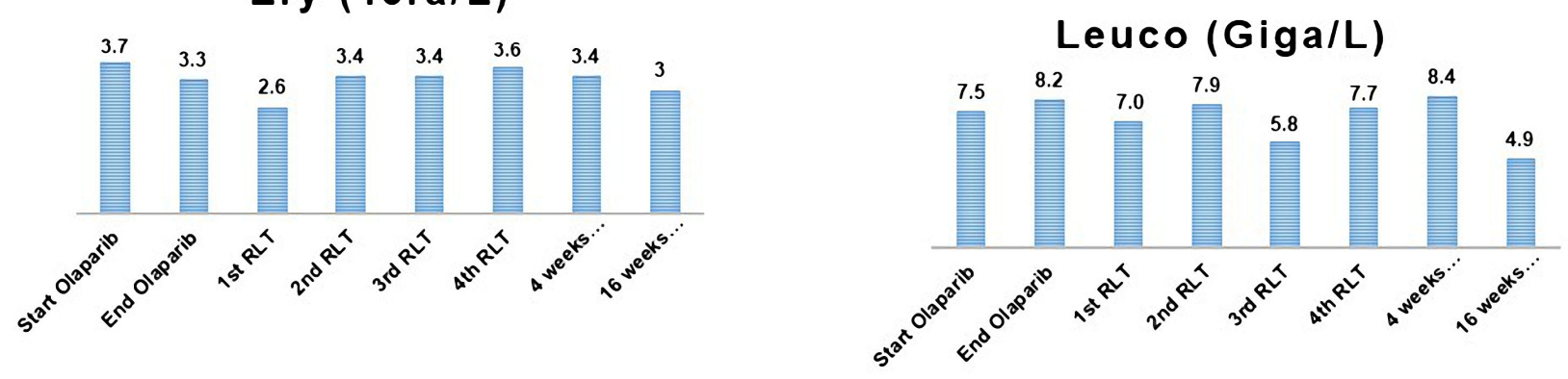

Figure 4: Laboratory values of patient 2 


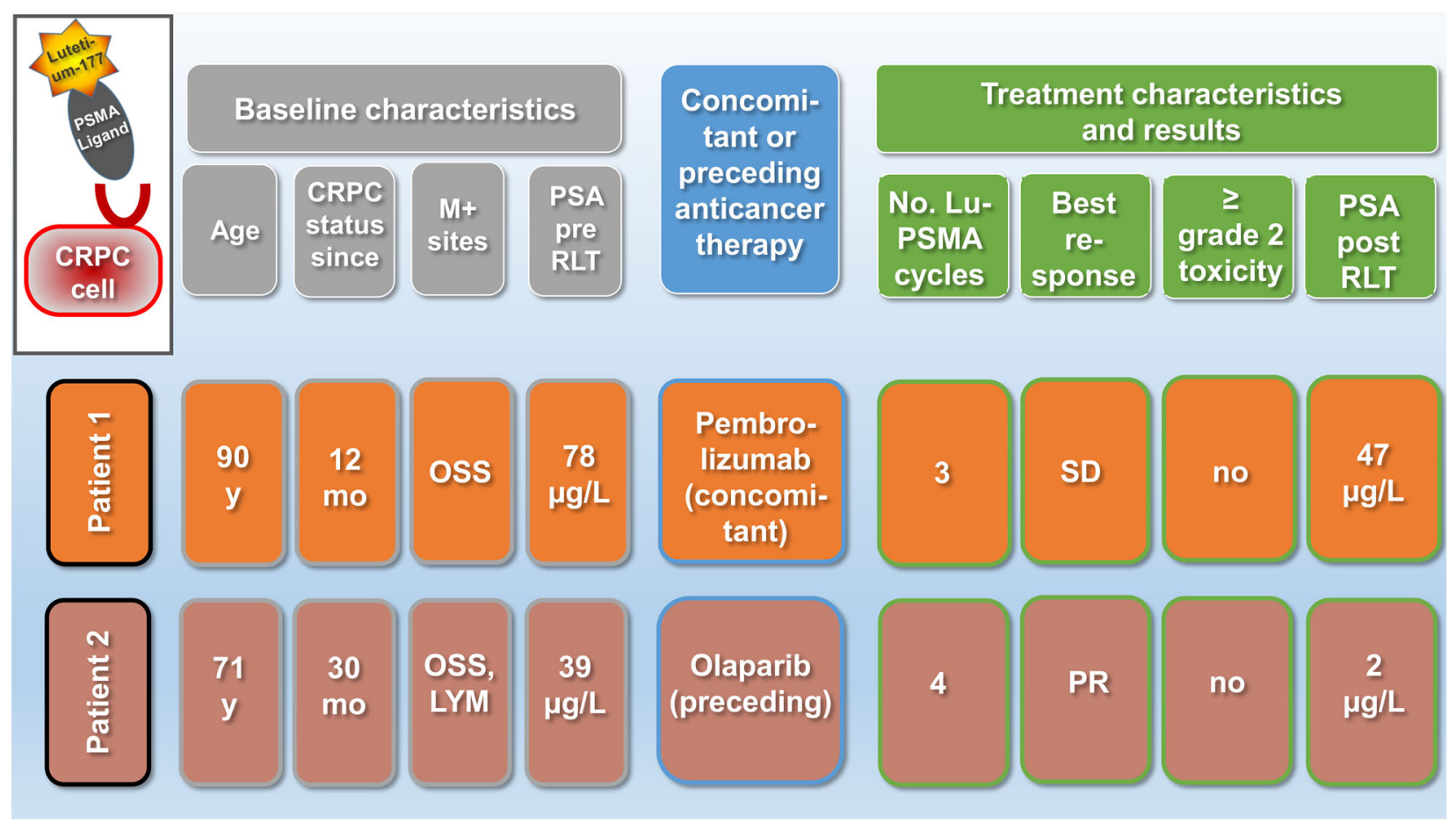

Graphical Abstract 